\title{
Construction of 1-Resilient Boolean Functions with Optimal Algebraic Immunity and Good Nonlinearity
}

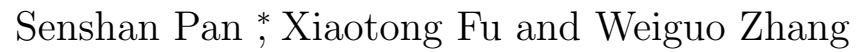 \\ ISN Lab, Xidian University, Xi'an 710071, P.R.China.
}

May 9, 2010

\begin{abstract}
This paper presents a construction for a class of 1-resilient Boolean functions with optimal algebraic immunity on an even number of variables by dividing them into two correlation classes, i.e. equivalence classes. From which, a nontrivial pair of functions has been found by applying the generating matrix. For $n$ is small (e.g. $n=6$ ), a part of these functions achieve almost optimal nonlinearity. Apart from their good nonlinearity, the functions reach Siegenthaler's [16] upper bound of algebraic degree. Furthermore, a class of 1-resilient functions on any number $n>2$ of variables with at least sub-optimal algebraic immunity is provided.
\end{abstract}

Keywords: Stream ciphers, Boolean functions, 1-Resilient, Algebraic immunity, Algebraic degree.

\section{Introduction}

Boolean functions are used as nonlinear combiners or nonlinear filters in certain models of stream cipher systems. Nowadays, a mounting number of attacks (Berlekamp-Massey attack, correlation attack, fast algebraic attack, i.e. FAA, etc.) has come out. This reality makes people have to revise old methods or design new ones to resist as many attacks as possible at the same time. Balance, a high nonlinearity, a high algebraic immunity, and in the case of the combiner model, a proper correlation immunity (in the case of the filter model, a correlation immunity of order 1 is commonly considered as sufficient) are the cryptographic characteristic of good stream ciphers. The interaction of them is so complex that some properties are contrary to others to some extend. For instance, Maiorana-McFarland construction together with its variations is a popular and favorable approach for a number of well-behaved functions so far. Being constructed by affine subfunctions, M-M construction, however, has an evident drawback against FAA [4]. To find the functions satisfying all the good characteristic by a trial and error method is unfeasible or at least harder and harder. It seems that in [4], a class of 1-resilient and optimal algebraic immunity functions was first obtained through a doubly indexed recursive relation. But its low nonlinearity impedes the utilization in cryptographic models. The construction employing symmetric functions present a risk if attacks using this peculiarity can be found in the future. Recently, [18] has provided 1-resilient

*Corresponding address: ISN Lab, Xidian University, Xi'an 710071, P.R.China. Email: pansenshan@gmail.com 
functions with maximum degree and optimal algebraic immunity by a primary construction, when the number of variables $n$ only equals to $6,8,10,12$. Bars and Viola in [1] are trying to find a complete combinatorial characterization and thence to good random generation algorithms for well-behaved functions. Being a first step towards their extremely tough direction, the work of [1] is interesting and admiring.

In this paper we propose a construction method to design 1-resilient Boolean functions on even number variables $(n \geq 3)$, which retain properties of the maximum degree and optimal algebraic immunity as the ones in [20]. The constructions provided in Section 3 reveal a good adaptability: a function with higher nonlinearity can be obtained merely by finding the base function with improved nonlinearity without of the change of generating methods. Besides, using the best example in [20], we find a part of the functions with almost optimal nonlinearity.

The organization of this paper is as follows. In Section 2, the basic concepts and notions are presented. In Section 3, we present a secondary construction (i.e. Siegenthaler's construction) by concatenating two balanced Boolean functions $f, g$ with odd variables $n$, where $\operatorname{deg}(f)=n-1$, $A I(f)=(n+1) / 2, g \in \hat{H}_{f}$ (cf. section 2). Our concrete realization is given in Section 4 by introducing the functions in [20]. In Section 5, a larger class of functions with sub-optimal algebraic immunity on any number $(\geq 2)$ of variables. Finally, section 6 concludes the paper.

\section{Preliminary}

A Boolean function $f(x)$ is a function from $\mathbb{F}_{2}^{n}$ to $\mathbb{F}_{2}$, where $x=\left(x_{1}, \cdots, x\right) \in \mathbb{F}_{2}^{n}$ and $\mathbb{F}_{2}^{n}$ is the vector space of tuples of elements from $\mathbb{F}_{2}$. To avoid confusion with the additions of integers in $\mathbb{R}$, denoted by + and $\Sigma_{i}$, we deliberately denote the additions over $\mathbb{F}_{2}$ by $\oplus$ and $\bigoplus_{i}$ for the purpose of arousing readers' attention. $f(x)$ is generally represented by its algebraic normal form (ANF):

$$
f(x)=\bigoplus_{u \in \mathbb{F}_{2}^{n}} \lambda_{u}\left(\prod_{i=1}^{n} x_{i}^{u_{i}}\right)
$$

where $\lambda_{u} \in \mathbb{F}_{2}, u=\left(u_{1}, \cdots, u_{n}\right)$. The algebraic degree of $f(x)$, denoted by $\operatorname{deg}(f)$, is the maximal value of $w t(u)$ such that $\lambda_{u} \neq 0$, where $w t(u)$ denotes the Hamming weight of $u$.

We denote $L T(f)=c t$ as the leading term of $f, L M(f)=t$ the leading monomial and $L C(f)=c$ the leading coefficient, where $c \in \mathbb{F}_{2}$ and $t$ is a monomial of $\mathbb{F}_{2}\left[x_{1}, \cdots, x_{n}\right] /\left(x_{1}^{2}+x_{1}, \cdots, x_{n}^{2}+x_{n}\right)$. Apparently, $L C(f)=1$ and $L T(f)=L M(f)=t$ as $f \in \mathbb{F}_{2}^{n}$. $f$ is called an affine function when $\operatorname{deg}(f)=1$. An affine function with constant term equal to zero is called a linear function. Any linear function on $\mathbb{F}_{2}^{n}$ is denoted by:

$$
\omega \cdot x=\omega_{1} x_{1} \oplus \cdots \oplus \omega_{n} x_{n}
$$

where $\omega=\left(\omega_{1}, \cdots, \omega_{n}\right), x=\left(x_{1}, \cdots, x_{n}\right) \in \mathbb{F}_{2}^{n}$. The Walsh spectrum of $f \in \mathcal{B}_{n}$ in point $\omega$ is denoted by $W_{f}(\omega)$ and calculated by

$$
W_{f}(\omega)=\sum_{x \in \mathbb{F}_{2}^{n}}(-1)^{f(x) \oplus \omega \cdot x} .
$$

$f \in \mathcal{B}_{n}$ is said to be balanced if its output column in the truth table contains equal number of 0's and 1's (i.e. $W_{f}(0)=0$ ). 
In [19], a spectral characterization of resilient functions has been presented.

Lemma 1: A $n$-variable Boolean function is $m$-resilient if and only if its Walsh transform satisfies

$$
W_{f}(\omega)=0, \text { for } 0 \leq w t(\omega) \leq m, \omega \in \mathbb{F}_{2}^{n} .
$$

The Hamming distance between two $n$-variable Boolean functions $f$ and $\rho$ is denoted by

$$
d(f, \rho)=\left\{x \in \mathbb{F}_{2}^{n}: f(x) \neq \rho(x)\right\} .
$$

The set of all affine functions on $\mathbb{F}_{2}^{n}$ is denoted by $A(n)$. The nonlinearity of a Boolean function $f \in \mathcal{B}_{n}$ is its distance to the set of all affine functions and is defined as

$$
N_{f}=\min _{\rho \in A(n)}(d(f, \rho)) .
$$

In term of Walsh spectra, the nonlinearity of $f$ is given by [10]

$$
N_{f}=2^{n-1}-\frac{1}{2} \cdot \max _{\omega \in \mathbb{F}_{2}^{n}}\left|W_{f}(\omega)\right|
$$

Parseval's equation [9] states that

$$
\sum_{\omega \in \mathbb{F}_{2}^{n}}\left(W_{f}(\omega)\right)^{2}=2^{2 n}
$$

So any Boolean function $f$ with $n$ variables satisfies

$$
\max _{\omega \in \mathbb{F}_{2}^{n}}\left|W_{f}(\omega)\right| \geq 2^{n / 2}
$$

the functions for which equality holds are called bent functions. Obviously, the nonlinearity of bent functions is $2^{n-1}-2^{n / 2-1}$, where $n$ is even.

Definition 1: Let $f$ be a Boolean function with $n$ variables. Then $f$ is said to be almost optimal if $N_{f} \geq 2^{n-1}-2^{(n-1) / 2}$ when $n$ is odd, and $N_{f} \geq 2^{n-1}-2^{n / 2}$ when $n$ is even.

Let $\operatorname{Supp}(f)=\left\{b_{i}=\left(b_{i 1}, \cdots, b_{i n}\right) \mid f\left(b_{i}\right)=1,1 \leq i \leq w t(f)\right\}$. Then $f$ can be represented as follows:

$$
f\left(x_{1}, \cdots, x_{n}\right)=\sum_{i=1}^{w t(f)} \prod_{j=1}^{n}\left(x_{j}+1+b_{i j}\right) .
$$

Clearly, $\operatorname{deg}(f)<n$ if and only if $w t(f)$ is even. Moveover, $\operatorname{deg}(f)=n-1$ if and only if wt(f) is even and

$$
\bigoplus_{i=1}^{w t(f)}\left(b_{i 1}, \cdots, b_{i n}\right) \neq 0
$$


Definition 2: The algebraic immunity $A I(f)$ of a n-variable Boolean function $f \in \mathbb{F}_{2}^{n}$ is defined to be the lowest degree of nonzero functions $g$ such that $f g=0$ or $(f+1) g=0$.

Definition 3: Let $n$ be the number of variables, a Boolean function with $n$ variables $f$ belongs to the correlation class $M_{f}$ defined by

$$
<w t(f), \operatorname{deg}(f), A I(f) ; \delta_{n}, \delta_{n-1}, \cdots, \delta_{1}>
$$

where $w t(f)$ is its Hamming weight, $\operatorname{deg}(f)$ is the algebraic degree of $f, A I(f)$ algebraic immunity and $\delta_{i}=w t\left(\left.f\right|_{x_{i}=0}\right)-w t\left(\left.f\right|_{x_{i}=1}\right)$, for any $1 \leq i \leq n$.

Definition 4: Let $f, g$ are two Boolean functions with n variables. The equivalence relation $\mathcal{R}$ is defined by

$$
f \mathcal{R} g \Longleftrightarrow M_{f}=M_{g}
$$

Generally, $<w t(f), \operatorname{deg}(f) ; \delta_{n}, \cdots, \delta_{1}>\left(<w t(f) ; \delta_{n}, \cdots, \delta_{1}>\right)$ is the correlation class without of the considering the algebraic immunity (the algebraic immunity and degree).Notice that, for $1 \leq j \leq n$, we can get $\delta_{j}=\sum_{i=1}^{w t(f)}(-1)^{b_{i j}}=w t(f)-2 b_{* j}$, where $b_{* j}=\sum_{i=1}^{w t(f)} b_{i j}$. Thus a simple conclusion can be reached as follows:

Proposition 1: For two Boolean functions $f$ and $g, \operatorname{deg}(f) \geq n-1$. If $g \in M_{f}$, then $L T(g)=L T(f)$, i.e. $f$ and $g$ have the same leading term.

Definition 5: Let $p, q \in 0, \cdots, 2^{n}, \zeta^{0}=<p ; \delta_{n}^{0}, \cdots, \delta_{1}^{0}>, \zeta^{1}=<q ; \delta_{n}^{1}, \cdots, \delta_{1}^{1}>$. The operator class $*$ is defined by

$$
\zeta^{0} * \zeta^{1}=\zeta
$$

where

$$
\zeta=<p+q ; \delta_{n+1}=p-q, \delta_{n}=\delta_{n}^{0}+\delta_{n}^{1}, \cdots, \delta_{1}=\delta_{1}^{0}+\delta_{1}^{1}>.
$$

Let $\zeta^{0} \times \zeta^{1}$ denote the set

$$
\left\{h \in\{0,1\}^{2^{n+1}} \mid h=f \| g=\left(1+x_{n+1}\right) f+x_{n+1} g, f \in \zeta^{0}, g \in \zeta^{1}\right\} .
$$

The following Lemma in [1] enable to decompose correlation classes recursively.

Lemma 2 (Decomposition):

$$
\zeta=\bigcup_{\zeta^{0} * \zeta^{1}=\zeta} \zeta^{0} \times \zeta^{1}
$$

Definition 4: Let $\zeta=<m, d, a i ; \delta_{n}, \delta_{n-1}, \cdots, \delta_{1}>$. The mirror class of $\zeta$ is the class

$$
\hat{\zeta}=<m, d, a i ;-\delta_{n},-\delta_{n-1}, \cdots,-\delta_{1}>
$$


An $r^{\text {th }}$ order Reed-Muller code $R(r, n)$ is the set of all binary strings(vectors) of length $2^{n}$ associated with the Boolean polynomials $f\left(x_{1}, x_{2}, \cdots, x_{n}\right)$ of degree at most $\mathrm{r}$. The collection of the Boolean functions with the leading term $L T(f)$ consists of the coset $f+R(\operatorname{deg}(f)-1, n)$.

Notation 1:

$$
\begin{aligned}
& H_{f}=M_{f} \bigcap(f+R(\operatorname{deg}(f)-1, n)), \\
& \hat{H}_{f}=\hat{M}_{f} \bigcap(f+R(\operatorname{deg}(f)-1, n)),
\end{aligned}
$$

where $\mathrm{n}$ is the number of variables of $f$.

So, Proposition 1 can be written as $g \in H_{f}$, if $\operatorname{deg}(f) \geq n-1$ and $g \in M_{f}$.

Definition 6: Let $f$ be a Boolean function with $n$ variables and Hamming weight $2 m$. Then, $\mathrm{f}$ is first-order correlation-immune when $w t\left(\left.f\right|_{x_{i}=0}\right)=w t\left(\left.f\right|_{x_{i}=1}\right)=m$, for any $1 \leq i \leq n$.

It is easily seen that $\zeta=\hat{\zeta}$ if and only if $\forall f \in \zeta$ is a first-order correlation-immune. Besides, $f \in \zeta \Leftrightarrow \hat{f} \in \hat{\zeta}$, where $\hat{f}=f(x \bigoplus 1)$ denotes the reverse of the string $f$. As a consequence, the two classes have the same cardinality: $|\zeta|=|\hat{\zeta}|$.

Proposition 2: The algebraic degree, algebraic immunity and nonlinearity of a Boolean function $f$ are invariant under an affine transformation towards its input (i.e. $g(x)=f(A x \bigoplus b)$, where $A \in G L_{n}\left(\mathbb{F}_{2}\right)$ and $\left.b \in \mathbb{F}_{2}^{n}\right)$.

\section{Degree Optimized 1-Resilient Functions with Optimal Algebraic Immunity}

Proposition 3 [4]: Let $f, g$ be two Boolean functions on the variables $x_{1}, x_{2}, \cdots, x_{n}$ with $A I(f)=$ $d_{1}$ and $A I(g)=d_{2}$. Let $h=\left(1+x_{n+1}\right) f+x_{n+1} g \in \mathbb{F}_{2}^{n+1}$. Then

1) If $d_{1} \neq d_{2}$, then $A I_{n+1}(h)=\operatorname{mind}_{1}, d_{2}+1$.

2) If $d_{1}=d_{2}=d$, then $d \leq A I_{n+1}(h) \leq d+1$, and $A I_{n+1}(h)=d$ if and only if there exists $f_{1}, g_{1} \in \mathbb{F}_{2}^{n}$ of algebraic degree $d$ such that $f * f_{1}=0, g * g_{1}=0$ or $(1+f) * f_{1}=0,(1+g) * g_{1}=0$ and $\operatorname{deg}\left(f_{1}+g_{1}\right) \leq d-1$.

Construction 1: Let $n$ be any odd integer such that $n \geq 3$ and $f$ is a balanced Boolean function with maximum degree $n-1$ and optimal algebraic immunity $(n+1) / 2$, i.e. $f \in<2^{n-1}, n-$ $1,(n+1) / 2 ; \delta_{n}, \cdots, \delta_{1}>$. Let

$$
h=\left(1+x_{n+1}\right) f+x_{n+1} g \in \mathbb{F}_{2}^{n+1},
$$

where $g \in \hat{H}_{f}$.

Notice $\hat{H}_{f}$ is not empty for any Boolean function $f$ aforementioned because of $\bar{f}=f+1 \in \hat{H}_{f}$. Besides, there is another trivial element $\hat{f}$ in $\hat{H}_{f}$. 
Theorem 1: $h \in \mathbb{F}_{2}^{n+1}$ in Construction 1 is 1-resilient Boolean function with maximum degree and optimal algebraic immunity, if $g \in \hat{M}_{f}$.

Proof: If $g \in \hat{M}_{f}$, due to Proposition $1, f$ and $g$ have the same leading term of degree $n-1$ (i.e. $\left.g \in \hat{H}_{f}\right)$ and $h$ is the concatenation of $f$ and $g, \operatorname{deg}(h) \leq n-1$. Besides, $h$ contains the monomial $L T(f)$, so we have $h \in<2^{n}, n-1, A I(h) ; 0,0, \cdots, 0>$, which is 1-resilient function of optimized degree. Using Proposition 3, $(n+1) / 2 \leq A I(h) \leq(n+3) / 2$ for $A I(f)=A I(g)=(n+1) / 2$. However, $A I(h)$ is upper bounded by $(n+1) / 2$, so $h$ has maximum algebraic immunity $(n+1) / 2$. Thus $h \in<2^{n}, n-1,(n+1) / 2 ; 0,0, \cdots, 0>$.

Theorem 2: The nonlinearity of $h$ in Construction 1 is $N_{h} \geq N_{f}+N_{g}$.

Proof: Let $x=\left(x^{\prime}, x_{n+1}\right), \omega=\left(\omega^{\prime}, \omega_{n+1}\right) \in \mathbb{F}_{2}^{n+1}$.

$$
\begin{aligned}
W_{h}(\omega) & =\sum_{x \in \mathbb{F}_{2}^{n+1}}(-1)^{\omega \cdot x \oplus h(x)} \\
& =\sum_{x \in \mathbb{F}_{2}^{n+1}}(-1)^{\omega^{\prime} \cdot x^{\prime} \oplus \omega_{n+1} x_{n+1} \oplus\left(1+x_{n+1}\right) f\left(x^{\prime}\right) \oplus x_{n+1} g\left(x^{\prime}\right)} \\
& =\sum_{x^{\prime} \in \mathbb{F}_{2}^{n}}(-1)^{f\left(x^{\prime}\right) \oplus \omega^{\prime} \cdot x^{\prime}}+(-1)^{\omega_{n+1}} \sum_{x^{\prime} \in \mathbb{F}_{2}^{n}}(-1)^{g\left(x^{\prime}\right) \oplus \omega^{\prime} \cdot x^{\prime}} \\
& =W_{f}\left(\omega^{\prime}\right)+(-1)^{\omega_{n+1}} W_{g}\left(\omega^{\prime}\right)
\end{aligned}
$$

$\operatorname{By}(4)$, we have

$$
N_{h} \geq N_{f}+N_{g}
$$

In particular,for $g=\bar{f}$ or $\hat{f}, N_{h}=2 N_{f}$.

Next, we want to figure out whether there is an nontrivial function in $\hat{H}_{f}$ (i.e. $g \neq \bar{f}, \hat{f}$ ). This can convert to proofing whether there is a third element in $H_{f}$ besides $f$ and $\hat{f}+1$. The answer seems yes, but it has not been proofed yet. So we leave it as an open problem.

However, another property is enough:

Proposition 4: A pair of Boolean functions with $\mathrm{n}$ variables $\left(f^{*}, g^{*}\right)$ deduced from a given $f \in \mathbb{F}_{2}^{n}$ can always be found, where $f$ is defined in Construction $1, \operatorname{deg}\left(f^{*}\right)=n-1, A I\left(f^{*}\right)=$ $(n+1) / 2, N_{f^{*}}=N_{f}$ and $g^{*} \in \hat{H}_{f^{*}}, g^{*} \neq \hat{f}, \bar{f}$.

Proof: Let us consider the affine transformations: $f(x) \mapsto f(A x \bigoplus b)$, where $A \in G L_{n}\left(\mathbb{F}_{2}\right)$ and $b \in \mathbb{F}_{2}^{n}$.

Recall $\operatorname{Supp}(f)=\left\{b_{i}=\left(b_{i 1}, \cdots, b_{i n}\right) \mid f\left(b_{i}\right)=1,1 \leq i \leq w t(f)=2^{n-1}\right\}$, where $b_{i}<b_{j}$ means

$$
b_{i k}<b_{j k}, b_{i k+1}=b_{j k+1}, b_{i k+2}=b_{j k+2}, \cdots, b_{i n}=b_{j n}, \exists 1 \leq k \leq n .
$$

Let $\left(n, 2^{n-1}\right)$ matrix $S_{f}=\left(b_{1}, b_{2}, \cdots, b_{2^{n-1}}\right) \cdot \operatorname{rank}\left(S_{f}\right) \leq n$, and any two columns of $S_{f}$ are distinct. Therefore, its rank is $n$, or else there must be a $k$, s.t. $b_{1 k}=b_{2 k}=\cdots=b_{2^{n-1} k}$, which indicates $f_{0}=$ $0, f_{1}=1$ or $f_{0}=1, f_{1}=0$ for $f=\left(1+x_{k}\right) f_{0}+x_{k} f_{1}$, where $f_{0}, f_{1} \in F_{2}\left[x_{1}, \cdots, x_{k-1}, x_{k+1}, \cdots, x_{n}\right]$. 
But the latter case is contrary to $\operatorname{deg}(f)=n-1$, where $n \geq 3$. $S_{f}$ can be regarded as a generating matrix and all of its codewords consist of the space of dimension $n$. Therefore, there are $2^{n}$ distinct codewords. The weight distribution of them denotes $\left\{w_{0}, w_{1}, \cdots, w_{2^{n-1}}\right\}$, so at least $2^{n-1}$ pairs of codewords has the same weight.

It can be seen that

$$
<\delta_{n}(f), \cdots, \delta_{1}(f)>\neq<0, \cdots, 0>
$$

because of Siegenthaler's upper bound.

1) If there is a $t$, s.t. $1 \leq t \leq n, \delta_{t}=0$, then $f\left(x \bigoplus 1_{t}\right) \in H_{f}$ and $f\left(x \bigoplus 1_{t}\right) \neq f(x)$, where $1_{t} \in \mathbb{F}_{2}^{n}$ denotes all of its coordinates are 0 except $t^{t h}$. Because there $\exists k, 1 \leq k \leq n$ and $k \neq t$, $\sum_{i=1}^{w t(f)} b_{i k}$ is odd from (6),

$$
\operatorname{Supp}\left(f\left(x \bigoplus 1_{t}\right)\right) \neq \operatorname{Supp}(f) .
$$

Clearly, $f\left(x \oplus 1_{t}\right) \neq \hat{f}+1$. Thus we can choose $\left(f(x), f\left(x \oplus 1_{t}\right)+1\right)$ as a nontrivial pair $\left(f^{*}, g^{*}\right)$.

2) If all $\delta \neq 0$. If there exists $\delta_{s}=\delta_{t}$, where $1 \leq s<t \leq n$. $s^{\text {th }}$ row differs from $t^{t h}$ row. A permutation matrix $P$ can be used to swap $x_{s}$ and $x_{t}$. Although $S_{f(P x)} \neq S_{f}$, a special case, $\operatorname{Supp}(f(P x))=\operatorname{Supp}(f)$ may be happen. In case of that situation, we can perform an invertible transformation to alter the rows of $S_{f}$ except $s^{t h}$ and $t^{t h}$ rows. Thus an nontrivial pair of $\left(f^{*}, g^{*}\right)$ can be obtained. If no two $\delta s$ are the same, a invertible matrix $A$ of dimension n may be employed to renew the generating matrix to $S_{f(A x)}$, which has two different codewords of the same weight. Similarly, we can obtain a required $\left(f^{*}, g^{*}\right)$.

\section{Concrete Realization}

This section presents a concrete realization using Boolean functions in [20] as $f$, which construction is as follows:

Construction 2 [20]: $f(x)$ denotes a Boolean function on $\mathbb{F}_{2}^{n}$ and $S u p p(f)=\left\{B^{i} b_{1} \mid 0 \leq\right.$ $\left.i \leq 2^{n-1}\right\}$, where $0 \neq b_{1} \in \mathbb{F}_{2}^{n}, B$ is the companion matrix of a primitive polynomial $p(x)=$ $x^{n}+c_{n-1} x^{n-1}+\cdots+c_{1} x+1$ over the field $\mathbb{F}_{2}$, i.e.

$$
B=\left(\begin{array}{ccccc}
0 & 0 & \ldots & 0 & 1 \\
1 & 0 & \ldots & 0 & c_{1} \\
\ldots & \ldots & \ldots & \ldots & \ldots \\
0 & 0 & \cdots & 1 & c_{n-1}
\end{array}\right)
$$

Theorem 3[20]: $f$ has maximum degree $\mathrm{n}-1$ and algebraic immunity $\lceil n / 2\rceil$. Besides, it reaches a high nonlinearity, which is better than [5].

Now, a class of 1-resilient Boolean functions which is still degree maximized and algebraic immunity optimized has been possessed by using $f$ aforementioned.

Example 1: Let $f$ denote a Boolean function on $\mathbb{F}_{2}^{5}$ from Construction 2 and $S u p p(f)=$ $\left\{B^{i} b_{1} \mid 0 \leq i \leq 2^{4}\right\}$, where $b_{1}=(1,0,0,0,0)^{T} \in \mathbb{F}_{2}^{5}$. When $p(x)=x^{5}+x^{2}+1, f$ has nonlinearity 10 . 
By choosing $g=f(A x \bigoplus b)+1, b=(1,0,0,0,0)^{T}$ and

$$
A=\left(\begin{array}{lllll}
1 & 1 & 0 & 0 & 0 \\
0 & 1 & 0 & 0 & 0 \\
0 & 0 & 1 & 0 & 0 \\
0 & 0 & 0 & 1 & 0 \\
0 & 0 & 0 & 0 & 1
\end{array}\right)
$$

we can get a 1-resilient function $h \in \mathbb{F}_{2}^{6}, A I(h)=3, \operatorname{deg}(h)=4$ and $N_{h}=2^{5}-2^{3}=24$, which is almost optimal. The following is the truth table of $h$ :

$$
6 D A 6 C 82 D 52953 B D 2 .
$$

Remark: The nonlinearity of $h$ is determined by the based functions $f$ and $g$, which is not almost optimal in [20] for a large $n$. So it is hard to obtain $h$ with almost optimal nonlinearity on large variables so far. Fortunately, we may achieve this goal by employing base functions with better nonlinearity in the future.

\section{1-Resilient Functions with Sub-Optimal Algebraic Immunity}

Generally, we can get a extended version of Construction 1 for any $n \geq 2$. This class of Boolean functions can achieve sub-optimized algebraic immunity.

Construction 3: let $n$ be any integer such that $n \geq 2$ and $f$ is a balanced Boolean function with maximum degree $n-1$ and optimal algebraic immunity $\lfloor(n+1) / 2\rfloor$, i.e. $f \in<2^{n-1}, n-1,(n+$ $1) / 2 ; \delta_{n}, \cdots, \delta_{1}>$. Let

$$
h=\left(1+x_{n+1}\right) f+x_{n+1} g \in \mathbb{F}_{2}^{n+1},
$$

where $g \in \hat{H}_{f}$.

Theorem 4: $h \in \mathbb{F}_{2}^{n+1}$ in Construction 3 is 1-resilient Boolean function with maximum degree and algebraic immunity at least $\lfloor(n+1) / 2\rfloor$, if $g \in \hat{M}_{f}$.

Example 2: We use the function $f \in \mathbb{F}_{2}^{16}$, where $N_{f}=32556$ [20], then $h$ is 1-resilient function with sub-optimal algebraic immunity and $N_{h} \geq 65112$.

\section{Conclusion}

In this paper, we have described a technique for constructing a class of 1-resilient functions with maximum degree and optimal algebraic immunity on even number variables. Unfortunately, this construction only results a part of the entire functions belong to $<2^{n}, n-1,(n+1) / 2 ; 0,0, \cdots, 0>$. Because it has other subsets

$$
\begin{array}{r}
<2^{n-1}, n-1,(n+1) / 2 ; \delta_{n}, \cdots, \delta_{1}>\times<2^{n-1}, n-1,(n-1) / 2 ;-\delta_{n}, \cdots,-\delta_{1}> \\
<2^{n-1}, n-2,(n+1) / 2 ; \delta_{n}, \cdots, \delta_{1}>\times<2^{n-1}, d,(n+1) / 2 ;-\delta_{n}, \cdots,-\delta_{1}>
\end{array}
$$


where $n \geq 3$ is odd, $d<n-2$. That is, $g$ do not have to be an affine transformation of $f$ towards input $x$. The characteristic of those classes are so far hard to predict. The best nonlinearity of Construction 1 is unknown except for a small number of variables. Hence we gain almost optimal functions $h$ with 6 variables. The adaptability of Construction 1 enable us to get the functions with a higher nonlinearity by introducing balanced functions $f$ with better nonlinearity than [20] in the future. In the end, we present a larger class of 1-resilient Boolean functions with sub-optimal Algebraic immunity.

\section{References}

[1] J.-M Le Bars and A. Viola, "Equivalence classes of Boolean functions for first-order correlation," IEEE Transactions on Information Theory, vol. 56, no. 3, pp. 1247-1261, Mar, 2010.

[2] P. Camion, C. Carlet, P. Charpin, and N. Sendrier, "On correlation-immune functions," in Advances in Cryptology - CRYPTO'91 (Lecture Notes in Computer Sceince). Berlin, Germany: Springer-Verlag, 1992, vol. 547, pp. 86-100.

[3] C.Carlet, "On bent and highly nonlinear balanced / resilient functions and their algebraic immunities," Proceedings of AAECC 16, LNCS 3857, pp. 1-28, 2006.

[4] C. Carlet, D. K. Dalai, K. C. Gupta and S. Maitra, "Algebraic immunity for cryptographically significant Boolean functions: Analysis and construction," IEEE Transactions on Information Theory, vol. 52, no. 7, pp. 3105-3121, Jul, 2006.

[5] C.Carlet and K.Feng, "An infinite class of balanced functions with optimal algebraic immunity, good immunity to fast algebraic attacks and good nonlinearity," in Proc. Advances in Cryptology-ASIACRYPT, Nerlin, Germany, 2008, vol. 5350, Lecture Notes in Computer Science, pp 425-440.

[6] S. Chee, S. Lee, D. Lee and S. H. Sung, "On the correlation immune functions and their nonlinearity," in Advances in CryptologyAsiacrypt'96 (Lecture Notes in Computer Sceince). Berlin, Germany: Springer-Verlag, 1997, vol. 1163, pp. 232-243.

[7] D. K. Dalai, K. C. Gupta, S. Maitra, "Notion of algebraic immunity and its evaluation related to fast algebraic attacks," In Second International Workshop on Boolean Function Cryptography and Applications, 2006.

[8] J. F. Dillon, Elementary Hadamard difference set, Ph.D. Thesis, University of Maryland, 1974.

[9] F.J. MacWilliams and N.J.A. Sloane, The Theory of Error-Correcting Codes, Amsterdam, The Netherlands: North-Holland, 1977.

[10] W. Meier and O. Staffelbach, "Nonlinearity criteria for cryptographic functions," in Advances in Cryptology - EUROCRYPT'89 (Lecture Notes in Computer Sceince), Berlin, Germany: Springer-Verlag, 1990, vol. 434, pp. 549-562.

[11] O. S. Rothaus, On 'bent' functions, Journal of Combinatorial Theory, Ser.A, vol. 20, pp. 300-305, 1976. 
[12] P. Sarkar and S. Maitra, "Construction of nonlinear Boolean functions with important cryptographic properties," in Advances in Cryptology - EUROCRYPT 2000 (Lecture Notes in Computer Sceince), Berlin, Germany: Springer-Verlag, 2000, vol. 1807, pp. 485-506.

[13] P. Sarkar and S. Maitra, "Nonlinearity bounds and constructions of resilient Boolean functions," in Advances in Cryptology - CRYPTO 2000 (Lecture Notes in Computer Sceince), Berlin, Germany: Springer-Verlag, 2000, vol. 1880, pp. 515-532.

[14] P. Sarkar and S. Maitra, "Construction of nonlinear resilient Boolean functions using small affine functions," IEEE Transactions on Information Theory, vol. 50, no. 9, pp. 2185-2193, Sept. 2004.

[15] J. Seberry, X.M. Zhang, and Y. Zheng, "On constructions and nonlinearity of correlation immune Boolean functions," in Advances in Cryptology - EUROCRYPT'93 (Lecture Notes in Computer Sceince), Berlin, Germany: Springer-Verlag, 1984, vol. 765, pp. 181-199.

[16] T. Siegenthaler, "Correlation-immunity of nonlinear combining functions for cryptographic applications," IEEE Transactions on Information Theory, vol. 30, no.5, pp. 776-780, September 1984 .

[17] Y. V. Tarannikov, "On resilient Boolean functions with maximum possible nonlinearity," in Progress in Cryptology - INDOCRYPT 2000 (Lecture Notes in Computer Science). Berlin, Germany: Springer Verlag, 2000, vol. 1977, pp. 19-30.

[18] Ziran Tu and Yingpu Deng, "A class of 1-resilient function with high nonlinearity and algebraic immunity," Cryptography ePrint Archive, Report 2010/179, 2010. http://eprint.iacr.org/.

[19] G.-Z. Xiao and J. L. Massey, "A spectral characterization of correlation-immune combining functions," IEEE Transactions on Information Theory, vol. 34, no. 3, pp. 569-571, 1988.

[20] Qichun Wang, Jie Peng, Haibin Kan and Xiangyang Xue. "Constructions of cryptographiclly significant Boolean functions using primitive polynomials," Will appear in IEEE Transactions on Information Theory, Vol. 56,No. 6, June, 2010. 